\title{
Left Atrium as an Active Component of the Pathophysiology in $\mathrm{HCM}$
}

\author{
Toshiyuki Ko, ${ }^{1} \mathrm{MD}$
}

(Int Heart J 2018; 59: 906-908)

$\mathrm{H}$ ypertrophic cardiomyopathy (HCM) is a genetic cardiomyopathy that causes left ventricular hypertrophy (LVH) and myocardial fibrosis. ${ }^{1)} \mathrm{HCM}$ is a disorder without a distinct ethnic or gender pattern of distribution. Its prevalence is estimated at $0.16-0.29 \%$ (1: 625-1:344 individuals) in the general population. ${ }^{2-5)}$ The screening of HCM is usually conducted by echocardiography, but when more sensitive imaging methods or genetic testing are used, a much higher estimate of $0.6 \%$ (1:167) has been reported. ${ }^{6,7)}$

Left ventricular outflow tract obstruction (hypertrophic obstructive cardiomyopathy; HOCM) in HCM patients is known to be associated with a high incidence of heart failure, various arrhythmias such as atrial fibrillation (AF), thromboembolic events, and sudden death. ${ }^{8)}$ Since the signature of HOCM is very characteristic, it is easily diagnosed, and the risk stratification and treatment are also well established. ${ }^{9)}$ Compared to HOCM, patients without ventricular obstruction (non-HOCM) usually have normal systolic function and are thought to have a stable clinical course. ${ }^{10)}$ However, some non-HOCM patients also experience heart failure, AF, and even death because of diastolic dysfunction and high LV filling pressures. Identifying non-HOCM patients with a poor prognosis is a major unmet need in clinical settings.

The left atrium (LA) acts as a reservoir during systole (LA reservoir function reflects the ability of the LA to fill), as a conduit during early diastole (LA conduit function reflects the contribution of the LA to early LV filling), and as an active pump during late diastole (LA booster function reflects LA contractile function). The thin left atrial wall is sensitive to changes in LV filling pressures. Therefore, left atrial function is a strong marker of the severity and chronicity of diastolic dysfunction. ${ }^{11,12}$ Many studies have described the diagnostic and prognostic importance of LA size and pressure both in the general population as well as in selected clinical conditions such as heart failure with preserved ejection fraction $(\mathrm{HFpEF}){ }^{12-15)}$

\begin{abstract}
Article p.991
In this issue of International Heart Journal, Shin, et al. examined the prognostic impact of LA minimal volume (LAVmin) on clinical outcome in a total of 167 consecutive non-HOCM patients. ${ }^{16)}$ They found LAVmin was an independent risk factor for composite outcome (HF hospitalization, stroke, mortality) in patients with nonHOCM. They also found that LAVmin was superior to LAVmax, which has previously been reported as an important indicator of left ventricular diastolic function and predictor of clinical outcome in HCM patients. ${ }^{17)}$ The result that LAVmin is superior to LAVmax in non-HOCM patientsis result is reasoned because early in 2012, Russo, et al. reported that LAVmin measured by real-time 3D echocardiography is more strongly correlated to LV diastolic function (E/e') and LV filling pressure than LAVmax. ${ }^{12}$ This suggests that LAVmin may be a good marker of LA remodeling caused by LV diastolic function. One of the important limitations of the study by Shin, et al. is that they used 2D echocardiography instead of 3D echocardiography. Previous studies have shown that $3 \mathrm{D}$ echocardiography is more accurate for the measurement of LA volume than 2D echocardiography. ${ }^{18,19)}$ Therefore, if we use 3D echocardiography in non-HOCM patients, LAVmin may have a prognostic impact and may be useful for risk stratification, even in the very early stage of the disease.

The modalities and technologies for the assessment of LA pathophysiology are rapidly advancing (Figure). In addition to $3 \mathrm{D}$ imaging of LA using echocardiography or cardiovascular magnetic resonance, echocardiographic speckle-tracking strain is also a technology in the spotlight because it can noninvasively measure the functional components of the LA, including reservoir, conduit, and contractile strain. LA reservoir strain, in particular, has strong prognostic value in HFpEF. In their study about the prognostic utility of LA strain, Freed, et al. reported that abnormal indices of LA mechanics (particularly LA reservoir strain) are powerful clinical and prognostic factors in HFpEF. $^{20)}$ In HCM patients, Fujimoto, et al. have shown
\end{abstract}

From the ${ }^{1}$ Department of Cardiovascular Medicine, Graduate School of Medicine, The University of Tokyo, Japan.

Address for correspondence: Toshiyuki Ko, MD, Department of Cardiovascular Medicine, Graduate School of Medicine, The University of Tokyo, 7-3-1 Hongo, Bunkyo-ku, Tokyo 113-8655, Japan. E-mail: tkou-tky@umin.ac.jp

Received for publication July 19, 2018. Revised and accepted August 2, 2018.

doi: $10.1536 /$ ihj. $18-441$

All rights reserved by the International Heart Journal Association. 


\section{Pathogenesis}

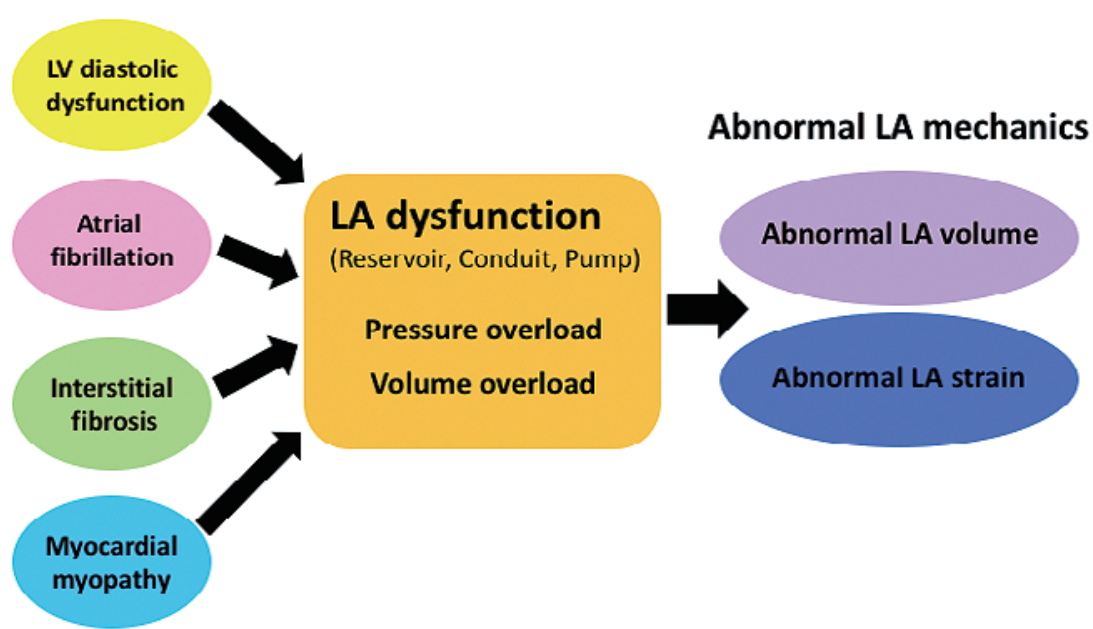

Figure. Pathogenesis of HCM and LA dysfunction. Various pathogeneses and abnormalities are observed in HCM, and induce LA dysfunction which can be detected by echocardiography and cardiovascular magnetic resonance.

that loss of LA active function (evaluated by LA strain) was independently associated with increased cardiac events. $^{21)}$ When recent insights about LA physiology and function are taken together, it creates a paradigm shift that the LA is no longer simply being a passive marker of disease severity, but an active component of the heart failure in HCM patients. Several questions still remain to be answered. Can LA dysfunction identify non-HOCM patients with a poor prognosis at an early stage, before they develop more overt forms of heart failure? And which parameter is suitable for the assessment of early LA dysfunction?

Although the striking pathologic features of the LV in HCM patients have attracted our attention for decades, it is now time to focus more on the LA. After we increase our understanding of the characteristics and pathophysiology of the LA, unloading therapy of LA and improvement of LA function may be important future therapeutic targets in HCM patients.

\section{Disclosures}

Conflicts of interest: None.

\section{References}

1. Marian AJ, Braunwald E. Hypertrophic Cardiomyopathy: Genetics, Pathogenesis, Clinical Manifestations, Diagnosis, and Therapy. Circ Res 2017; 121: 749-70.

2. Maron BJ, Gardin JM, Flack JM, Gidding SS, Kurosaki TT, Bild DE. Prevalence of hypertrophic cardiomyopathy in a general population of young adults. Echocardiographic analysis of 4111 subjects in the CARDIA Study. Coronary Artery Risk Development in (Young) Adults. Circulation 1995; 92: 785-9.

3. Maron BJ, Mathenge R, Casey SA, Poliac LC, Longe TF. Clinical profile of hypertrophic cardiomyopathy identified de novo in rural communities. J Am Coll Cardiol 1999; 33: 1590-5.

4. Hada Y, Sakamoto T, Amano K, et al. Prevalence of hy- pertrophic cardiomyopathy in a population of adult Japanese workers as detected by echocardiographic screening. Am J Cardiol 1987; 59: 183-4.

5. Zou Y, Song L, Wang Z, et al. Prevalence of idiopathic hypertrophic cardiomyopathy in China: a population-based echocardiographic analysis of 8080 adults. Am J Med 2004; 116: 14-8.

6. Bick AG, Flannick J, Ito K, et al. Burden of rare sarcomere gene variants in the Framingham and Jackson Heart Study cohorts. Am J Hum Genet 2012; 91: 513-9.

7. Maron BJ, Peterson EE, Maron MS, Peterson JE. Prevalence of hypertrophic cardiomyopathy in an outpatient population referred for echocardiographic study. Am J Cardiol 1994; 73: 577-80.

8. Autore C, Bernabo P, Barilla CS, Bruzzi P, Spirito P. The prognostic importance of left ventricular outflow obstruction in hypertrophic cardiomyopathy varies in relation to the severity of symptoms. J Am Coll Cardiol 2005; 45: 1076-80.

9. Geske JB, Ommen SR, Gersh BJ. Hypertrophic Cardiomyopathy: Clinical Update. JACC Heart Fail 2018; 6: 364-75.

10. Maron MS, Rowin EJ, Olivotto I, et al. Contemporary Natural History and Management of Nonobstructive Hypertrophic Cardiomyopathy. J Am Coll Cardiol 2016; 67: 1399-409.

11. Teo SG, Yang H, Chai P, et al. Impact of left ventricular diastolic dysfunction on left atrial volume and function: a volumetric analysis. Eur J Echocardiogr 2010; 11: 38e43.

12. Russo C, Jin Z, Homma S, et al. Left atrial minimum volume and reservoir function as correlates of left ventricular diastolic function: impact of left ventricular systolic function. Heart 2012; 98: 813-20.

13. Gottdiener JS, Kitzman DW, Aurigemma GP, Arnold AM, Manolio TA. Left atrial volume, geometry, and function in systolic and diastolic heart failure of persons $>$ or $=65$ years of age (the cardiovascular health study). Am J Cardiol 2006; 97: 83-9.

14. Kurt M, Wang J, Torre-Amione G, Nagueh SF. Left atrial function in diastolic heart failure. Circ Cardiovasc Imaging 2009; 2: $10-5$.

15. von Roeder M, Rommel KP, Kowallick JT, et al. Influence of left atrial function on exercise capacity and left ventricular function in patients with heart failure and preserved ejection fraction. Circ Cardiovasc Imaging 2017; 10: e005467.

16. Shin SH, Jang JH, Baek YS, et al. Prognostic impact of left atrial minimal volume on clinical outcome in patients with non- 
obstructive hypertrophic cardiomyopathy. Int Heart J 2018; 59: 991-5.

17. Losi MA, Betocchi S, Barbati G, et al. Prognostic significance of left atrial volume dilatation in patients with hypertrophic cardiomyopathy. J Am Soc Echocardiogr 2009; 22: 76-81.

18. Mor-Avi V, Yodwut C, Jenkins C, et al. Real-time 3D echocardiographic quantification of left atrial volume: multicenter study for validation with CMR. JACC Cardiovasc Imaging 2012; 5: 769-77.

19. Badano LP, Miglioranza MH, Mihăilă S, et al. Left atrial volumes and function by three-dimensional echocardiography: Ref- erence values, accuracy, reproducibility, and comparison with two-dimensional echocardiographic measurements. Circ Cardiovasc Imaging 2016; 9: e004229.

20. Freed BH, Daruwalla V, Cheng JY, et al. Prognostic utility and clinical significance of cardiac mechanics in heart failure with preserved ejection fraction: Importance of left atrial strain. Circ Cardiovasc Imaging 2016; 9: e003754.

21. Fujimoto K, Inoue K, Saito M, et al. Incremental value of left atrial active function measured by speckle tracking echocardiography in patients with hypertrophic cardiomyopathy. Echocardiography 2018 (in press). 